\title{
FOLLOW-UP COMO MODALIDADE DA ASSISTÊNCIA DE ENFERMAGEM AO HIPERTENSO*
}

Fátima Lúcia Machado Braga** Cristiane Maria Covello***

\begin{abstract}
RESUMO: O presente trabalho analisa o papel da enfermeira na equipe multidisciplinar da Clínica de Hipertensão Arterial do Hospital das Clínicas da Universidade Federal de Pernambuco e sua atuação junto a clientes hipertensos controlados apenas com o follow-up da enfermagem, através da consulta específica. As autoras descrevem a metodologia e os resultados obtidos na consulta de enfermagem, ressaltando a característica de autonomia profissional nos procedimentos terapêuticos estabelecidos no protocolo da Clínica. Além disso, são analisadas as variações das cifras tensionais dos hipertensos controlados, a necessidade de encaminhamento ao médico e/ou a outros profissionais da equipe, o cumprimento da terapêutica farmacológica e/ou não famacológica, o comportamento dos fatores de risco modificáveis identificados na primeira consulta ou nas consultas subseqüentes e a adesão ao follow-up de enfermagem.
\end{abstract}

ABSTRACT: The following study analyses the nurse's role in a multiprofessional working group at the hypertension ambulatory care unit (Hospital das Clínicas Universidade Federal de Pernambuco, Brasil) and her role in working with hypertensive patients, who are managed only by the nursing follow-up at the clinic. The authors describe the methodology and the results obtained from the management of these patients at the clinic, emphasizing the practitioner's autonomy in the therapeutic procedures, which are established in the clinic's protocol. Other aspects are also analysed in this study such as (1) the blood pressure variations of a controlled group of hypertensive patients, (2) the necessity of referring the patient to a physician or other professionals when needed, (3) the results of pharmacologiacal and/or non-pharmacological therapeutics and (4) the influences of the modifying risk factors, which were identified either in the first consultation or in subsequent clinic follow-up, and the aderence to the nursing follow-up.

UNITERMOS: Consulta de Enfermagem - Hipertenso Controlado - Hipertensão Arterial.

\section{INTRODUÇÃO}

As doenças cardiovasculares (DCV) são responsáveis, atualmente, pela maioria dos óbitos registrados no Brasil. Entre os fatores de risco cardiovasculares, a hipertensão arterial (HA) é considerada de maior relevância por sua alta prevalência e importância social, pois atinge 10 a $20 \%$ da população adulta em idade produtiva, com reflexos extremamente negativos na

Trabalho apresentado no $45^{\circ}$ Congresso Brasileiro de Enfermagem. Prêmio Lays Netto dos Reis - $1^{\circ}$ Lugar. Olinda-Recife, 28 de novembro a 3 de dezembro de 1993.

** Enfermeira, Especialista em Enfermagem de Saúde Pública. Sanitarista do Hospital das Clínicas (HC) - Universidade Federal de Pernambuco (UFPE). Gerente de Enfermagem do Serviço Ambulatorial de Cardiologia/Nefrologia e enfermeira da equipe multidisciplinar da Clínica de Hipertensăo Arterial do HC-UFPE.

*** Enfermeira. Residente do Hospital Regional Dom Moura e estagiária da Clínica de Hipertensáo Arterial do HC-UFPE. 
situação sócio-político-econômica do país(3, 4, $15,28)$.

O Ministério da Saúde (MS), diante das tendências atuais e levando em conta a magnitude do problema da HA no contexto geral das doenças cardiovasculares, orienta a integração interinstitucional e multidisciplinar em todos os niveis de assistência (primária, secundária ou terciária), seja no plano individual ou coletivo. As medidas preventivas são valorizadas, pois causam maior impacto na redução da morbi-mortalidade das DCV associadas à esta afecção.(4)

Coerente com esse pensamento, o Programa Nacional de Educação e Controle da Hipertensão Arterial (PNECHA) foi criado em 1985 e vem desenvolvendo ações coordenadas nas áreas de pesquisa, educação e assistência, procurando delinear o perfil epidemiológico da HA e fundamentar os procedimentos, técnicas e métodos individuais e coletivos(4).

A esta filosofia de trabalho, integrou-se também a Universidade Federal de Pernambuco (UFPE). Assim, no Serviço de Cardiologia do Hospital das Clínicas (HC) da UFPE, a partir de junho de 1990, foi criada a Clínica de Hipertensão Arterial (CHA), apoiando-se nos principios defendidos pelo MS. (4)

Considerando ser a abordagem multidisciplinar um elemento facilitador no controle da pressão arterial (PA) e na adesão ao tratamento(12, $22,42,43)$, desde $o$ início dos trabalhos foi formada uma equipe constituída por um médico, uma enfermeira, uma assistente social, uma nutricionista e uma auxiliar de enfermagem, cujo atendimento é demonstrado em fluxograma próprio (Anexo 1).

O relevante papel da enfermeira nos programas de HA e na organização e execução de protocolos nos serviços especializados é comentado por vários autores $(10,17,18,19,39,45)$ e reconhecido oficialmente por organismos como o Joint National Committee on Detection, Evaluation and Treatment of High Blood Pressure (JNC) $(23,24)$ e o National High Blood Pressure Coordinating Committee e a National Conference on High Blood Pressure Control, quando incluem esse profissional em seus quadros. Concordando com essa opinião, BURKE(5) descreve diretrizes, normas e rotinas específicas que garantem maior autonomia clínica à enfermeira e asseguram a referência dos casos selecionados para atendimento médico. Além do mais, estes pesquisadores afirmam que a autonomia profissional confere maior responsabilidade e serve de estímulo, mantendo o interesse e satisfação da enfermeira no seu trabalho.

A importância do trabalho da enfermeira junto a hipertensos com terapêutica estabelecida e cifras pressóricas mantidas em níveis aceitávis (hipertenso controlado) é comentada por BURKE et al.(5), CLARK(10), CRUZ(13), GIBLIN(19), KOCHAR et al.(26), PIERIN(36, 37), SOUSA et al. $(42,43)$ e WARD et al. $(45)$. Os autores citam clínicas onde o hipertenso controlado fica sob a responsabilidade da enfermeira que, através da consulta de enfermagem (CE), se encarrega de monitorizar o tratamento e reforçar orientações (follow-up da enfermagem), referindo ao médico apenas aqueles com problemas que extrapolem sua competência resolutiva.

Embasado nessas opiniőes, o Programa preliminar elaborado pela equipe da CHA já incluia, entre as principais atividade de enfermagem a serem desenvolvidas, a CE a todos os clientes hipertensos, fossem novos ou subseqüentes $(1,2)$.

A primeira CE direcionada para as doenças cardiovasculares é realizada em formulário próprio (Anexos 2 e 3) e sistematizada de forma que as infor-mações registradas sirvam de subsídios aos outros membros da equipe. Esse procedimento foi adaptado do modelo de Wanda de Aguiar Horta(20) e incorpora, além das normas técnicas e operacionais do PNECHA(4), outras preconizadas pela $1^{\circ}$ Convenção Brasileira para o Tratamento da Hipertensão Arterial(11) e pelo J.N.C. $(23,24)$.

Para facilitar a execução das consultas subseqüentes e promover maior integração das atividades multiprofissionais, foi elaborado um formulário único, onde é registrado o atendimento de cada membro da equipe, adaptado do modelo utilizado na Liga de Hipertensão Arterial da Universidade Federal de Goiás.

Em novembro de 1992 ficou estabelecida uma nova modalidade de assistência de enfermagem. Desde então, o cliente em tratamento farmacológico e/ou não famacológico, com cifras pressóricas mantidas dentro dos parâmetros aceitáveis e com exame clínico/laboratorial satisfatório (hipertenso controlado), é liberado pelo médico para controle apenas com o follow- 
up da enfermagem. A partir desse momento, 0 cliente fica sob total responsabilidade da enfermeira, para acompanhamento clínico (clinical follow-up) que inclui a monitorização da PA em períodos de 60 a 90 dias e reforço das medidas higieno-dietéticas.

A cada retomo o cliente é reavaliado clinicamente e, quando detectado problema que transcenda às suas atribuições, a enfermeira o encaminha a outros membros da equipe e/ou a outros serviços.

Este trabalho analisa a atuação da enfermeira em equipe multidisciplinar, na assistência ao cliente hipertenso controlado apenas com o follow-up da enfermagem (hipertenso controlado).

\section{METODOLOGIA}

Foram estudados 261 clientes hipertensos, com pressão arterial (PA) mantida dentro de parâmetros aceitáveis (hipertenso controlado), matriculados na Clínica de Hipertensão Arterial (CHA) - Serviço de Cardiologia (SC) do Hospital das Clínicas (HC) - da Universidade Federal de Pernambuco (UFPE), acompanhados pela enfermeira através de consulta de enfermagem (CE), no período de novembro de 1992 a maio de 1993.

Da amostragem foram selecionados 126 clientes, de acordo com o protocolo estabelecido, levando em conta os seguintes aspectos:

1- o cliente com cifras tensionais controladas na segunda CE (primeira consulta subseqüente) deverá ser atendido também pelo médico, assistente social e nutricionista;

2- o cliente só poderá ser considerado controlado a partir da terceira consulta subseqüente;

3- O cliente hipertenso controlado deverá ter sido atendido pela enfermeira em, pelo menos, quatro vezes.

Foram excluídos da amostragem 135 clientes que não atendiam aos critérios pré-estabelecidos, sendo que, destes, 63 possuiam duas consultas de controle e 54 somente uma consulta. Também foram excluídos 18 clientes considerados faltosos no período em estudo.

Os dados foram coletados dos formulários padronizados da clínica e do Mapa de Registro Diário das Atividades da equipe. Para comple- mentação das informações foram consultados prontuários arquivados no Serviço de Arquivo Médico e Estatística (SAME) e os registros dos arquivos do Serviço Social e Serviço de Nutriçåo.

Ao final da avaliação clínica, com duração média de vinte minutos, o cliente era aprazado para retorno a cada 2/3 meses e orientado para comparecimento antes deste prazo, se surgissem intercorrências. O cliente poderia ainda ser referido ao médico, nutricionista e assistente social, quando detectados problemas especificos, inerentes a estes profissionais.

Considerou-se como faltoso o cliente que não compareceu para o controle após 3 meses da data de coleta de dados e que não tinha consulta agendada para outro período.

Os dados de comparecimento à CHA foram colhidos do Mapa de Registro Diário das Atividades, sendo computadas todas as consultas e faltas de clientes hipertensos controlados e nåo controlados, nos meses de novembro de 1992 a maio de 1993, inclusive aquelas que foram excluídas da população analisada.

Através de computação manual, os dados coletados foram dispostos em tabelas e gráficos, adotando-se como tratamento estatístico o emprego de números absolutos e relativos, com cálculo da média e desvio-padrão para as idades. Para facilitar os cálculos foi feito o arredondamento para a primeira casa decimal.

\section{Definição de Termos}

Adesão: é a assiduidade do cliente ao Programa nos dias previamente marcados pela enfermeira.

Clientes Novos: são os clientes que comparecem à Clínica de Hipertensão Arterial (CHA), pela primeira vez e são registrados no Programa, durante a primeira consulta de enfermagem.

Clientes Subsequientes: são os clientes que retomam à CHA, na data aprazada pela enfermeira, após a primeira consulta de enfermagem.

Follow-up de Enfermagem: acompanhamento do cliente hipertenso com pressão arterial controlada, com fármacos e/ou orientação higieno-dietética, pela enfermeira.

Hipertensos Controlados: clientes com cifras pres-sóricas mantidas, dentro de niveis 
Tabela 1: Encaminhamento dos hipertensos controlados pela enfermeira ao médico, nutricionista e assistente social, após a consulta de enfermagem - nov. 92/maio 93

\begin{tabular}{l|c|c|c|c|c|c|c}
\hline $\begin{array}{r}\text { Atendi- } \\
\text { Meses }\end{array}$ & $\begin{array}{c}\text { Consulta de } \\
\text { Enfermagem }\end{array}$ & \multicolumn{2}{c|}{ Consulta Médica } & \multicolumn{2}{c|}{ Consulta Nutriçăo } & \multicolumn{2}{c}{ Entrevista Serviço Social } \\
\cline { 2 - 9 } & $\mathrm{n}^{\circ}$ de consultas & $\mathrm{n}^{0}$ consultas & $\%^{*}$ & $\mathrm{n}^{\circ}$ consultas & $\%^{*}$ & $\mathrm{n}^{\circ}$ consultas & $\%^{*}$ \\
\hline novembro/92 & 57 & 14 & 24,0 & 32 & 56,0 & 22 & 38,6 \\
\hline dezembro/92 & 60 & 09 & 15,0 & 14 & 23,3 & 09 & 15,0 \\
\hline janeiro/93 & 41 & 07 & 17,0 & 12 & 29,2 & 03 & 7,3 \\
\hline fevereiro/93 & 61 & 04 & 6,5 & 15 & 24,5 & 14 & 22,9 \\
\hline março/93 & 49 & 03 & 6,1 & 19 & 38,8 & 04 & 8,2 \\
\hline abril/93 & 61 & 00 & 0,0 & 18 & 29,5 & 04 & 6,6 \\
\hline maio/93 & 45 & 05 & 11,1 & 26 & 57,8 & 02 & 4,4 \\
\hline TOTAL & 374 & 42 & 11,2 & 136 & 36,3 & 58 & 15,5 \\
\hline
\end{tabular}

Fonte: SAME - Hospital das Clinicas - UFPE

* Os percetuais foram calculados em relaçáo ao número de consultas de cada mês.

aceitáveis (literatura), cujo acompanhamento está sob a responsabilidade direta da enfermeira.

\section{APRESENTAÇÃO E ANÁLISE DE DADOS}

Será analisada a importância do follow-up da enfermagem no trabalho multidisciplinar da Clinica de Hipertensão Arterial (CHA), através dos dados coletados em relação à necessidade de encaminhamento aos outros profissionais da equipe, terapêutica indicada, fatores de risco modificáveis identificados e adesão ao Programa.

Os dados são apresentados sob a forma de tabelas e figuras, cuja análise se reporta à literatura consultada.

A tabela 1 revela que de um total de 374 hipertensos consultados pela enfermeira, apenas $42(11,2 \%)$ necessitaram de atendimento médico no periodo, sendo que, destes, 23 $(54,8 \%)$ obedecendo ao protocolo de serviço onde, após a primeira consulta subseqüente de enfermagem, é obrigatória a consulta médica e $19(45,2 \%)$, por necessitarem substituir as drogas em uso devido ao alto custo, ou por patologias intercorrentes.

A análise desses instrumentos confima a responsabilidade e autonomia da enfermeira no acompanhamento do grupo de hipertensos, liberados da assistência médica, enquanto o tratamento indicado obtiver o efeito desejado. Este é direcionado principalmente para o reforço das orientações e esclarecimentos, que assegurem a adesão ao tratamento e para o desenvolvimento de habilidades e atitudes do cliente visando o auto-cuidado.

Consideramos também que a metodologia da consulta de enfermagem (CE) utilizada na CHA favorece a avaliação clínica global do hipertenso, garantindo uma prescrição adequada e o encaminhamento seguro para os outros profissionais da equipe, quando a situação exigir.

Para CLARK e DUNN(10), GIBLIN(19) e KOCHAR e WOODS(26), a enfermeira, pelo seu conhecimento e habilidades, tem assumido maior nivel de responsabilidade não só na educação, mas também no follow-up de hipertensos controlados, orientada por protocolos claros e precisos. Segundo os autores, essa modalidade de assistência favorece não só o cliente, mas também o médico que pode dedicar mais tempo aos hipertensos mais graves, não controlados. Os autores citam clínicas independentes, administradas por enfermeiras, onde estas profissionais podem instruir terapia medicamentosa padronizada, ou ajustar aquelas já prescritas sob supervisão médica periódica. Nesse caso, a interação médico-enfermeira é fundamental e o cliente deverá tomar conhecimento de que o tratamento é feito em conjunto.

BURKE e CECIL(5), CAR et al.(6), CRUZ(13), JARDIM E SOUZA(21), PIERIN E CAR(34), SOU$S A$ et al. $(42,43)$ relatam experiências com followup, através da CE, a hipertensos com terapêutica já estabelecida pelo médico. A literatura consultada destaca dois aspectos importantes para o êxito do controle da pressão arterial (PA): a 
Tabela 2: Clientes hipertensos controlados pela enfermeira com medidas não farmacológicas, associadas ou não ao uso de drogas - nov 92/maio 93.

\begin{tabular}{l|c|c|c|c|c}
\hline \multirow{2}{*}{ Meses } & \multicolumn{5}{|c}{ Hipertensos Controlados } \\
\cline { 2 - 6 } & Total & \multicolumn{2}{|c}{$\begin{array}{c}\text { Medidas farmacológicas e } \\
\text { năo farmacológicas }\end{array}$} & \multicolumn{2}{c}{ Medidas nåo farmacológicas } \\
\cline { 2 - 6 } & $\mathrm{n}^{0}$ consultas & $\mathrm{n}^{0}$ consultas & $\%^{*}$ & $\mathrm{n}^{0}$ consultas & $\%^{*}$ \\
\hline novembro/92 & 57 & 42 & 73,7 & 15 & 26,3 \\
\hline dezembro/92 & 60 & 46 & 76,6 & 14 & 23,3 \\
\hline janeiro/93 & 41 & 30 & 73,1 & 11 & 26,8 \\
\hline fevereiro/93 & 61 & 48 & 78,7 & 13 & 21,3 \\
\hline març/93 & 49 & 38 & 77,5 & 11 & 22,4 \\
\hline abril/93 & 61 & 45 & 73,8 & 16 & 26,2 \\
\hline maio/93 & 45 & 36 & 80,0 & 09 & 20,0 \\
\hline TOTAL & 374 & 285 & 76,2 & 89 & 23,8 \\
\hline
\end{tabular}

Fonte: SAME - Hospital das Clínicas - UFPE

* os percentuais foram calculados em relaçáo ao número de consultas de cada mês

educação para a saúde com ênfase nas medidas não farmacológicas e direcionamento para o auto-cuidado.

A tabela 2 demonstra que, na maioria das consultas, $285(76,2 \%)$ dos clientes hipertensos mantiveram a PA controlada com medidas higieno-dietéticas e fármacos, contra $89(23,8 \%)$ hipertensos controlados apenas com medidas não farmacológicas.

Os dados acima destacam a importância do papel da enfermeira junto a este grupo de hipertensos, sob sua responsabilidade, pois, apesar de não indicar as drogas, ela está encarregada de monitorizar o tratamento e avaliar as reais condições de saúde do cliente a cada retomo. Para isso, deverá estar capacitada para reconhecer os efeitos colaterais indesejáveis das drogas, para identificar causas de alterações de PA com uso ou não de hipotensores e para detectar precocemente possiveis danos em órgãos-alvo. desta forma, poderá proceder os encaminhamentos aos outros componentes da equipe como segurança e estabelecer o tipo de orientação adequada.

Sobre o assunto, OLIVEIRA et al.(33) afirmam que a enfermeira ...é o elemento capacitado para planejar e prestar assistência de enfermagem, visando o controle dos niveis tensionais da população hipertensa.

A tabela 3 aborda os principais fatores de risco modificáveis envolvidos no surgimento ou agravamento a hipertensão arterial (HA). Com os dados nela apresentados analisaremos 0 comportamento destes fatores ao longo do follow-up da enfermagem, no período estudado.

Foram analisados cinco importantes fatores de risco (tabagismo, etilismo, estresse, sedentarismo e obesidade), todos passiveis de serem reduzidos e/ou suprimidos dos hábitos e estilo de vida dos clientes, através de um processo educativo contínuo, onde é valorizada a participação ativa do hipertenso e de sua família, e estimulada a assunção da responsabilidade conjunta com o seu tratamento.

Tabela 3: Comportamento dos principais fatores de risco modificáveis identificados na consulta de enfermagem, nov. 92/maio 93

\begin{tabular}{|c|c|c|c|c|c|c|c|c|c|c|}
\hline \multirow{2}{*}{$\begin{array}{l}\text { Fatores de Risco } \\
\text { Consultas }\end{array}$} & \multicolumn{2}{|c|}{ Tabagismo } & \multicolumn{2}{|c|}{ Etilismo } & \multicolumn{2}{|c|}{ Estresse } & \multicolumn{2}{|c|}{ Sedentarismo } & \multicolumn{2}{|c|}{ Obesidade } \\
\hline & $n^{0}$ & $\%^{*}$ & $n^{0}$ & $\%^{*}$ & $n^{0}$ & $\% *$ & $n^{0}$ & $\%^{*}$ & $n^{0}$ & $\% *$ \\
\hline $1^{2}$ consulta & 16 & 12,7 & 02 & 1,6 & 35 & 27,8 & 106 & 84,1 & 72 & 57,1 \\
\hline $1^{a}$ consulta controle & 16 & 12,7 & 01 & 0,8 & 47 & 37,3 & 70 & 55,6 & 75 & 59,5 \\
\hline $2^{a}$ consulta controle & 11 & 8,7 & - & - & 39 & 31,0 & 30 & 38,2 & 63 & 50,0 \\
\hline $3^{2}$ consulta controle & 06 & 4,8 & - & - & 21 & 16,7 & 33 & 26,2 & 54 & 42,9 \\
\hline
\end{tabular}

Fonte: SAME - Hospital das Clínicas - UFPE.

* Os percentuais foram calculados em relaçăo aos 126 clientes hipertensos selecionados da amostra. 
Apesar de terem sido excluídos de nossa análise, por dificuldade técnica em compararmos suas variações ao longo do acompanhamento, foram identificadas alterações lipídicas em $74(58,7 \%)$ dos clientes, intolerância à glicose em $35(27,8 \%)$, uso de contraceptivos de 1 $(0,8 \%)$ e hipertrofia ventricular esquerda em 41 $(32,5 \%)$.

No que diz respeito ao uso inadequado de sal e gorduras saturadas na alimentação, constatamos que a maioria $123(97,6 \%)$ dos hipertensos já fazia restrição e sódio e 107 (84,9\%) evitavam gorduras saturadas.

\section{Tabagismo}

Dos 126 clientes estudados, 16 (12,7\%) referiram tabagismo na primeira $C E$, porém, quando comparados com a terceira consulta controle, verificamos que apenas $6(4,8 \%)$ clientes hipertensos persistiram com este hábito.

Apesar de vários estudos epidemiológicos não apontarem associação direta entre o fumo e a HA, mostram correlação estreita com a arteriosclerose que seria um fator agravante da doença hipertensiva. Os fumantes têm mais chance de evoluir para formas malignas da doença hipertensiva e apresentam incidência elevada de morte súbita(11, 15, 16, 17, 25, 26, 27, 34, 40, 41)

Apesar do pequeno número de fumantes em nossa clientela, nós não deixamos de enfatizar os maleficios deste vício em todas as oportunidades, seja no atendimento individual ou coletivo. Nessa ocasião, aproveitamos para elogiar os que conseguiram abolir o fumo e incentivar os fumantes a abandonarem o vício.

\section{Etilismo}

Como mostra a tabela 3 , um número insignificante de etilistas foi detectado na amostra, sendo $2(1,6 \%)$ na primeira $C E$, com uma redução de $100 \%$ quando comparado com a terceira consulta controle.

Ao contrário do tabagismo, o etilismo tem demonstrado intima ligação com a HA. Diversos ensaios têm indicado prevalência mais elevada de $\mathrm{HA}$ em individuos que consomem duas, três ou mais doses de álcool por dia, o que corresponde a $45 \mathrm{~g}$ ou mais de etanol $(5,26,27,28)$.
De acordo com URBANETZ(44), a PA aumenta linearmente com a elevação do consumo do álcool. A autora chama a tenção para o efeito reversivel após a desintoxicação, sendo que ...novas elevaçóes dos níveis pressoricos naqueles que voltam a beber, podem culminar em danos irreversíveis.

\section{Estresse}

Ao contrário do etilismo e tabagismo, um número considerável de nossa amostra $(35=27,8 \%)$ demonstrou este fator de risco na primeira CE. Estes índices se mostraram elevados na primeira $(47=37,3 \%)$ e segunda $(39=31 \%)$ consulta controle.

Consideramos que este fator requer um diagnóstico especializado que foge à nossa competência. Entretanto, a experiência tem nos mostrado que alguns clientes, durante as primeiras abordagens, podem apresentar sentimentos de ansiedade e até mesmo angústia. Provavelmente, esse comportamento se deve ao ambiente hospitalar e ainda ao tímido relacionamento coma enfermeira e os outros membros do grupo. Reforçando nossa hipótese, na terceira consulta controle observamos uma redução no número de clientes estressados para 21 (16,7\%).

KOCHAR e WOODS(26), LOLIO(27) e LUNA ${ }^{(28)}$ confirmam a influência do estresse na HA, bem como a dificuldade em mensurá-lo, e afirmam que sua diminuição influenciaria beneficamente a PA.

\section{Sedentarismo}

Este fator também mostrou-se elevado em nossa amostra. Na primeira CE obtivemos um total de $106(84,1 \%)$ registros e na terceira consulta controle, $33(26,2 \%)$. Comparando esses dados com os outros números da tabela, verificamos uma redução gradativa e acentuada deste fator de risco, durante o follow-up.

Embora o papel do exercício físico no tratamento da HA seja ainda bastante controverso, os indivíduos sedentários parecem ter risco $35 \%$ maiorde desenvolverem a doença, quando comparados com os que realizam exercícios regulares não isométricos ou aeróbicos $(7,14,16,26,28$, $30,31,44$ ). 


\section{Obesidade}

Como mostra a tabela 3, a obesidade foi um fator de risco importante presente em nossa amostra de 126 hipertensos, sendo que na primeira CE tivemos $72(57,1 \%)$ obesos, enquanto que na terceira consulta controle obtivemos 54 $(42,9 \%)$.

O comportamento insatisfatório desse fator no decorrer do nosso acompanhamento, devese, provavelmente, à dificuldade financeira para o cumprimento da dieta indicada pela nutricionista e à resistência do hipertenso para a mudança dos hábitos alimentares.

KOCHAR(26) chama a atenção para a dificuldade dos obesos assimilarem a necessidade da perda de peso e destacam a atuação da enfermeira nos programas educativos direcionados para esse fim. Segundo o autor, a enfermeira ...deve enfocar a responsabilidade do paciente pelo cuidado com seu próprio organismo.

Vários autores consideram a excessiva ingestão calórica o fator nutricional mais importante na patogênese da $H A$, e indicam a redução do peso como primeiro método de tratamento da doença hipertensiva $(7,11,18,29,30,41,44)$. CHAVES Jr et al. $(9,10)$, CRUZ $^{(14)}$, JARDIM e SOU$S A^{(21)}$ E OIGMAN(32) sugerem o envolvimento da equipe multiprofissional de clientes e familiares nesse processo de transformação. Para SOUSA et al. $(42,43)$ essa abordagem proporciona benefícios adicionais ao controle dos fatores de risco cardiovascular ...interferindo positivamente nas modificaçðes de hábitos alimentares e no modo de vida.

Acreditamos que a modalidade de assistência prestada na CHA garante a intervenção multiprofissional nos diversos aspectos biopsicossociais do nosso cliente. Respeitando sua individualidade, que inclui crenças, valores, motivaçăo e sentimentos verbalizados ou não, procuramos incentivá-lo a desenvolver atitudes que possam favorecer mudanças nos hábitos alimentares e no estilo de vida inadequados. 0 conhecimento sobre seu estado de saúde e a consciência de sua responsabilidade para o êxito do tratamento contribuirão decisivamente para a prevenção ou redução de danos ao organismo.
Tabela 4: Demostrativo do número de clientes hipertensos controlados e não controlados que compareceram a CHA-HC-UFPE nos meses de nov/92 a maio/93

\begin{tabular}{l|c|c|c|c}
\hline \multirow{2}{*}{$\begin{array}{c}\text { Clientes } \\
\text { Hipertensos }\end{array}$} & \multicolumn{2}{c|}{ Presentes } & \multicolumn{2}{c}{ Faltosos } \\
\cline { 2 - 5 } & $\mathrm{n}^{\circ}$ & $\%$ & $\mathrm{n}^{\circ}$ & $\%$ \\
\hline controlados & 733 & 70,3 & 71 & 31,0 \\
\hline năo controlados & 310 & 29,7 & 158 & 69,0 \\
\hline TOTAL & 1043 & 100,0 & 229 & 100,0 \\
\hline
\end{tabular}

Fonte: SAME - Hospital da Clínicas - UFPE

A tabela 4 evidencia que o número de hipertensos controlados $(733=70,3 \%)$ é duas vezes maior que o de năo controlados $(310=29,7 \%)$ e que o número de faltosos náo controlados (158=69\%) é o dobro dos faltosos hipertensos controlados $(71=31 \%)$.

Os dados apresentados sugerem que o follow-up de enfermagem, através da consulta sistematizada e direcionada para o auto-cuidado, contribui decisivamente para a adesão do cliente ao Programa. No entanto, faltam-nos dados consistentes para convalidar esta aderência.

CRUZ(14), KOCHAR e WOODS(26), OIGMAN(31,32), PIERIN e CAR(34), PIERIN et al. (35), PIERIN $(36,37,38)$, SOUSA et al. $(42,43)$ e WARD et al.(45), ressaltam a atuação da enfermeira como elemento catalizador das ações educativas, ocasião em que promove condições favoráveis ao esclarecimento de dúvidas, fortalecimento de hábitos de saúde, incentivo ao auto-cuidado e estímulo à aderência ao tratamento.

Rosenfeld e Silverberg in $\operatorname{PIERIN}(36,37)$ e in CAR et al.(6) revelam que a atuação da enfermeira no processo educativo aos hipertensos, integrada aos outros profissionais, elevou a taxa de controle de $39,2 \%$ para $72,8 \%$ e a de abandono foi reduzida de $30 \%$ para $0,65 \%$.

Estudo comparativo procedido por Rocha e Maginot in PIERIN(38), com dois grupos de hipertensos, mostra que, no primeiro grupo, seguido exclusivamente por médicos, os niveis pressóricos dos clientes se mantiveram inalterados e 0 indice de abandono foi de $38 \%$. No outro grupo acompanhado por equipe composta de enfermeiras, assistente social e nutricionais, as cifras tensionais foram reduzidas $11,9 \%$ e a taxa de abandono foi de $18 \%$.

Estas informações fundamentam nossa opinião e consolidam nossos achados.

Consideramos ser a abordagem multidisci- 
plinar um fator promocional no desenvolvimento da atenção holística, em que o cliente é visto como um ser humano biopsicossocial, especialmente os portadores de patologias crônicas que são influenciadas pelos fatores ambientais, como a hipertensão arterial.

\section{CONCLUSÃO}

Esta experiência pioneira no Hospital das Clínicas da Universidade Federal de Pernambuco abriu uma nova perspectiva de atuação da enfermeira em equipe multidisciplinar, favorecendo o controle dos clientes hipertensos e elevando o nível técnico desse profissional.

Ao concluir este trabalho, levantamos alguns aspectos que reforçam tais afirmações, quais sejam;

- os fatores de risco para a hipertensão arterial podem ser diminuídos e/ou suprimidos dos hábitos do cliente hipertenso controlado, pela enfermeira, através de processo educativo contínuo e direcionado para o auto-cuidado;

- a enfermeira com habilidades e conhecimentos técnico-cientícos especializados pode atuar na equipe multiprofissional, controlando os hipertensos com tratamento já estabeleci- do;

- o número de hipertensos controlados, pela enfermeira, que necessita de assistência médica é relativamente baixo, proporcionando mais tempo ao médico para atender os clientes com pressão arterial elevada e/ou com patologias concomitantes;

- essa modalidade de atendimento é eficazmente comprovada pela elevada aderência demonstrada na análise e,

- na equipe multidisciplinar, o cliente é tratado, considerando-se os aspectos biopsicossociais e a enfermeira está capacitada para encaminhá-lo a outros profissionais, quando as suas condições exigirem um atendimento especializado.

Esperamos que este trabalho possa conduzir as enfermeiras à uma reflexão, sobre a prática atual da enfermagem ambulatorial aos clientes portadores de doenças crônicas e degenerativas.

Sugerimos às enfermeiras que assumam sua posição de profissional liberal e autônoma, ocupando um espaço rico em perspectivas futuras, para que possamos contribuir eficaz e efetivamente para a promoção e proteção da saúde da população.

\section{REFERÊNCIAS BIBLIOGRÁFICAS}

1. BRASIL. Lei n 7498, de 25 de junho de 1986. Dispóe sobre a regulamentaçáo do exercicio da enfermagem e dá outras providéncias. Normas e Noticias - CO FEN.Brasília, a.10, p.3-5, jun. 1987. Ediçăo extra.

2. Decreto $n$ 94.406, de 08 de junho de 1987. Regulamenta a lei $n 7498$, de 25 de junho de 1986 , que dispóe sobre o exercício da enfermagem e dá outras providéncias. Normas e Noticias - CO FEN.Brasília, a.10, p.3-5, jun. 1987. Ediçăo extra.

3. Ministério da Saúde. Cademos das doenças crónico-degenerativas. Brasília: Divisăo Nacional de Doenças Crónico-Degenerativas, 1987. 22p.

4. . Normas Técnicas para o Programa Nacional de Educação e Controle da Hipertensão Arterial (PNECHA).Brasília: Centro de Documentaçăo do Ministério da Saúde, 1988. 90p.

5. BURKE, Eddie, CECIL, John. Hypertension: a practice nurse protocol. The Practitioner.s.1, v.235, n.1506, p.651-655, sep. 1991.

6. CAR, Márcia Regina, PIERIN, Ângela Maria Geraldo, AQUINO, Vera Lúcia. Estudo sobre a influência do processo educativo no controle da hipertensăo arterial. Rev. Esc. Enferm. USP. Săo Paulo, v.25, n.3, p.259-269, dez. 1991.

7. CERRATO, Paul L. Hypertension: the role of diet and lifestyle. R.N.s.1, v.53, n.12, p.46-51, dec. 1990.
8. CHAVES Jr., Hilton. Condutas da hipertensăo. Hiper Ativo. Sáo Paulo, a.1, n.6, p.3-6, maio 1992.

9. CHAVES Jr., Hilton, MACHADO, Fátima, VASCONCELOS, Ana Carolina, et al. Experiência multidisciplinar na Clínica de Hipertensăo do Hospital das Clínicas da Universidade Federal de Pernambuco. In: CONGRESSO DA SOCIEDADE BRASILEIRA DE CARDIOLOGIA, 48, 1992. Recife/Olinda. Anais... Recife/Olinda: Sociedade Brasileira de cardiologia, 1992. p.167.

10. CLARK, Anita B., DUNN, Marvin. A nurse clinician's role in the management of hypertensio. Arch. Inter. Med. s.1, v.136, n.8, p.903-904, aug. 1976.

11. CONVENÇÃO BRASILEIRA PARA O TRATAMENTO DA HIPERTENSÃO ARTERIAL, 1, 1990, Campos do Jordăo. Consenso brasileiro para o tratamento da hipertensăo arterial. Arq. Bras. Cardiol. Săo Paulo, v.56, s.A, p.A3-A9, jan. 1991.

12. COSTA, Maria José Chaves. Atuaçáo do enfermeiro na equipe multiprofissional. Rev. Bras. Enferm. Brasilia, v.31, p.321-339, jul./set. 1978.

13. CRUZ, Isabel Cristina Fonseca da. Consulta de Enfermagem ao Cliente Hipertenso. Rio de Janeiro, 90p., 1988. Dissertaçáo (Mestrado em Enfermagem Médico-Cirúrgica), Escola de Enfermagem Ana Néri, Universidade Federal do Rio de Janeiro, 1988. 
14. CRUZ, Diná Almeida Lopes Monteiro da. Aspectos de enfermagem na prevençăo primária das doenças isquêmicas do coraçăo. Rev. Esc. Enferm. USP.Sáo Paulo, v.22, n.2, p.215-221, ago. 1988.

15. ELUF NETO, José, LOTUFO, Paulo Andrade, LOLIO, Cecilia Amaro. Tratamento da hipertensăo arterial e declínio de mortalidade por acidentes vasculares cerebrais. Rev. Saúde Pública. Săo Paulo, v.24, n.4, p.332-336, 1990.

16. FELDER, Emma. Hypertension in blacks: implications for health care. The ABNF Joumal. s.1, v.2, n.1, p.11-14, Winter, 1991.

17. FEURY, Dianne, NASH, David T. Hypertension: the nurse's role. R.N.s.1, v.53, n.11, p.54-60, nov. 1990.

18. FINK, Janis $W$. The Challenge of high blood pressure control. Nursing Clinics of North America. s.1, v.16, n.2, p.301-308, june 1981.

19. GIBLIN, Elizabeth. Controling high blood pressure. American Joumal of Nursing. s.1, v.78, n.5, p.824, may 1978.

20. HORTA, Wanda Aguiar. Processo de Enfermagem.Sáo Paulo: EPU-Ed. da Universidade de Sáo Paulo, 1979. 100p.

21. JARDIM, Paulo César Veiga, SOUSA, Ana Luisa L. Liga de Hipertensáo Arterial integrando serviço, ensino e pesquisa. Boletin de la Oficina Sanitaria Panamericana.s.1, v.114, n.1, jan. 1993.

22. JARDIM, Paulo César Veiga, SOUSA, Ana Luisa L. Avaliaçăo da aderência ao tratamento da hipertensáo arterial em serviço multidisciplinar. In: Congresso da Sociedade Brasileira de Hipertensăo, 1, 1992. Såo Paulo. Anais... Sáo Paulo: Sociedade Brasileira de Hipertensăo, 1992.

23. JOINT NATIONAL COMMITTEE: The 1988 report of the Joint National Committee on Detection, Evaluation and Treatment of High Blood Pressure, 4. Arch. Intem. Med.v.148, p.1023-1238.

24. JOINT NATIONAL COMMITTEE: The 1992 report of the Joint National Committee on Detection, Evaluation and Treatment of High Blood Pressure. Ciéncia, Cultura e Lazer. Såo Paulo, a.1, n.1, p.12-13, 1993.

25. KIRK-GARDNER, Rhonda, CROSSMAN, Jane. Cardiac risk factors of smoking, hypertension, obesity and family history: a review of the literature. Can. J. Cardiovasc. Nurs.s.1, v.2, n.1, p.9-14. apr. 1991.

26. KOCHAR, Mahendr, WOODS, Karyn D. Controle da hipertensão para enfermeiras e demais profissionais de saúde.2.ed. Săo Paulo: ANDREI, 1990. 318p.

27. LOLIO, Cecília Amaro de. Prevalência da hipertensáo arterial em Ararquara. Arq. Bras. Cardiol.Sảo Paulo, v.55, n.3, p.167-173, set. 1990.

28. LUNA, Rafael Leite. Hipertensåo arterial.2.ed. Rio de Janeiro: MEDSI, 1990. 300p., p.1-74.

29. MARANHĀO, Mário Fernando Camargo, RAMIRES, Joáo António F. Aspectos atuais do tratamento da hipertensăo arterial. Arq. Bras. Cardiol.Sảo Paulo, v.51, n.1, p.99-105, jul. 1988.

30. MOSER, Marvin. Hypertension: how therapy works. American Joumal of Nursing. s.1, p.937-941, may 1990.

31. OIGMAN, Wille. Recomendaçőes năo famacológicas coadjuvantes no tratamento da hipertensáo arterial. Arq. Bras. Cardiol. Săo Paulo, v.57, n.4, p.341-346, out. 1991.
32. OIGMAN, Wille. Caminhos e descaminhos da hipertensăo. Galenus.Rio de Janeiro, v.1, n.2, p.5-10, 1991.

33. OLIVEIRA, Maria Ester Dias de, ERNESTO, Dayse $Z$. Lopez, CANCINO, Carola Angeles. Consulta de enfermagem a hipertensos: perfil da populaçăo e caracterizaçáo da assistência. Rev. Esc. Enferm. USP.Săo Paulo, v.22, n.2, p.189-198, ago. 1988.

34. PIERIN, Ângela Maria Geraldo, CAR, Márcia Regina. Instrumento de consulta de enfermagem a pessoas com hipertensáo arterial em tratamento ambulatorial. Rev. Esc. Enferm. USP. Sảo Paulo, v.26, n.1, p.1732, mar. 1992.

35. PIERIN, Ângela Maria G., CAR, Márcia Regina, GIORGI, Dante M.A. et al. Atendimento de enfermagem ao paciente com hipertensáo arterial. Rev. Bras. Med. (Cardiologia). Săo Paulo, v.3, n.4, agosto 1984.

36. PIERIN, Ângela Maria Geraldo. A pessoa com hipertensáo arterial em tratamento no ambulatório: consideraçơes gerais. Parte 1. Rev. Esc. Enferm. USP. Săo Paulo, v.22, n.2, p.223-229, ago. 1988.

37. PIERIN, Ângela Maria Geraldo. A pessoa com hipertensáo arterial em tratamento no ambulatório. Parte 2. Estudo sobre os problemas, dificuldades e modificaçóes decorrentes da doença e do tratamento. Rev. Esc. Enferm. USP Sáo Paulo, v.22, n.3, p.273-282, dez. 1988.

38. PIERIN, Ângela Maria Geraldo. A pessoa com hipertensâo arterial em tratamento no ambulatório. Parte 3. Influência de variáveis, conhecimento das complicaçóes da doença e expectativas quanto à assistência de enfermagem. Rev. Esc. Enferm. USP Sáo Paulo, v.23, n.1, p.35-48, abr. 1989.

39. RAMSAY, Janice A., McKENZIE, John K., FISH, David G. Physicians and nurse practitioners: do they provide equivalent health care? American Joumal of Public Health.s.1, v.72, n.1, p.55-57, jan. 1982.

40. ROCHA, Marisa M. Fatores de risco e coronariopatias. Medicina Prat-K. Rio de Janeiro, v.1, n.1, p.6-7, jan./fev. 1987.

41. SARAGOÇA, Manoel António. Tratamento da hipertensáo arterial. Rev. Ass. Med.Brasil. Sáo Paulo, v.38, n.3, p.159-168, jul./set. 1992.

42. SOUSA, Ana Luíza L., JARDIM, Paulo César V., MONEGO, Estelamaris Tronco et al. Uma experiência multiprofissional na abordagem ao paciente hipertenso. Arq. Bras. Cardiol. Såo Paulo, v.59, n.1, p.31-35, jul. 1992.

43. SOUSA, Ana Luiza L., JARDIM, Paulo César V., MONEGO, Estelamaris Tronco et al. A enfermagem e o paciente hipertenso em uma abordagem multidisciplinar. In: CONGRESSO BRASILEIRO DE ENFERMAGEM, 44, 1992, Brasília, Anais... Brasília: Associaçăo Brasileira de Enfermagem, 1992.

44. URBANETZ, Lorena A.G. Lara Teran. Hipertensăo arterial: mitos e verdades. Documenta Médica. Sáo Paulo, p.1-12, ago 1987.

45. WARD, Graham, BANDY, Patrícia, FINK, Janis W. Treating and counseling the hypertensive patient. American Joumal of Nursing. s.1, v.78, n.5, p.824-828, may 1978.

Recebido para publicaçăo em 20.12.93 
Fluxograma de Atendimento - Clínica de Hipertensão Arterial - HC - UFPE - 1992.

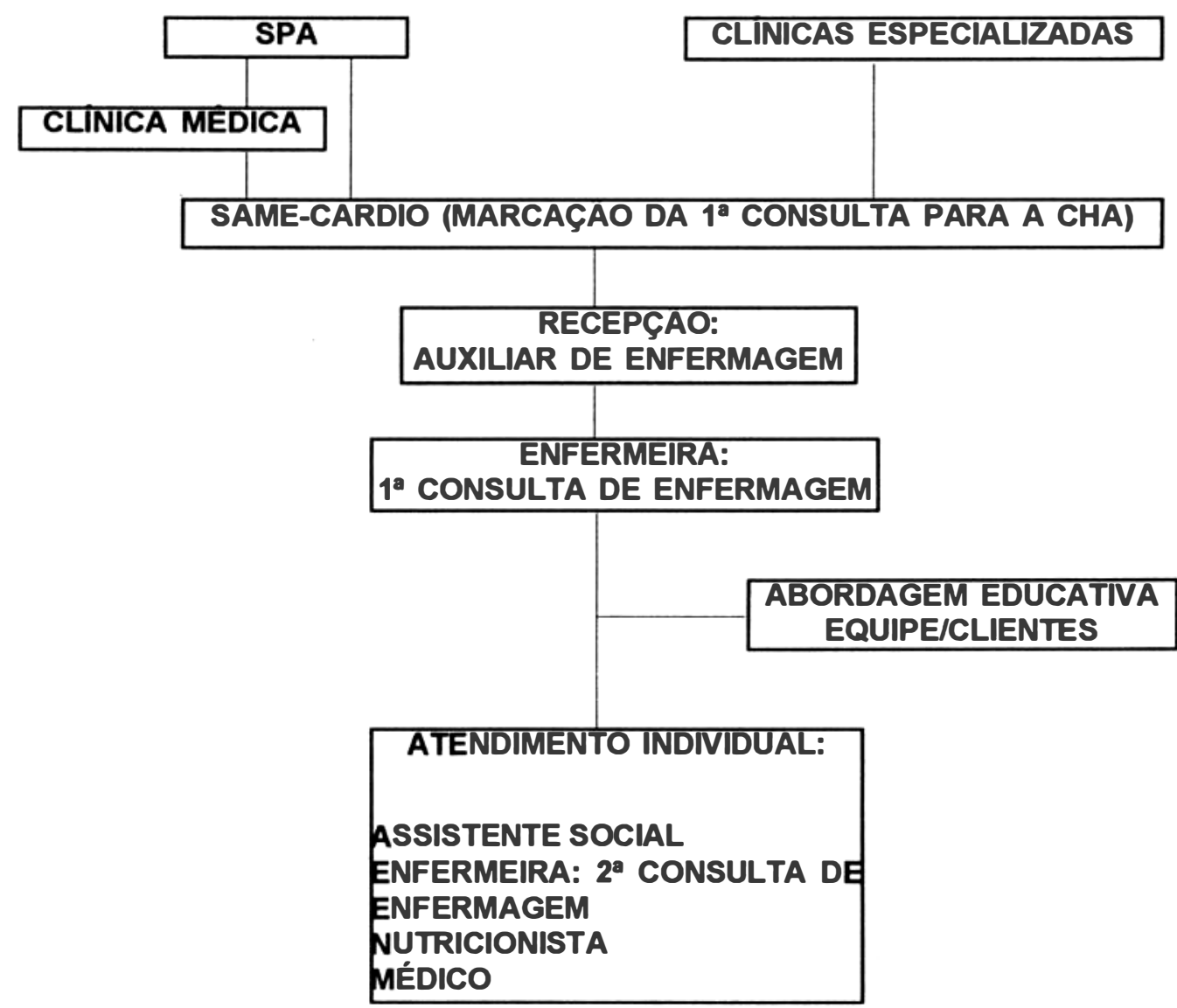




\section{ANEXO 2 \\ UNIVERSIDADE FEDERAL DE PERNAMBUCO \\ HOSPITAL DAS CLÍNICAS \\ SERVIÇO DE CARDIOLOGIA \\ CLÍNICA DE HIPERTENSÃO ARTERIAL}

CONSULTA DE ENFERMAGEM

1. DADOS DE IDENTIFICAÇÃO:

NOME

REGISTRO

IDADE SEXO

INSTRUÇÃO PROFISSÃO COR

DATA: ESTADO CIVIL OCUPAÇÃO

NATURALIDADE PROCEDÊNCIA RELIGIÃO

ENCAMINHADO POR ACOMPANHADO

2. QUEIXAS PRINCIPAIS:

CEFALÉIA ( ), TONTURA ( ), DISPNÉIA DE ESFORÇO: MÍNIMO ( ) MÉDIO ( ) GRANDE ( ), DPN ( ), PALPITAÇÃO ( ), EDEMA DE MMII ( ), DORPRECORDIAL ( ), ESCOTOMA ( ), INSÓNIA ( ), CÂIMBRA ( ), ASTENIA ( ), OUTROS:

3. HISTÓRIA DA DOENÇA ATUAL:

3.1 TRATAMENTO REALIZADO:

3.1.1 DIETA: NORMAL ( ) HIPOSSÓDICA ( ) OUTRAS:

3.1.2 DROGA HIPOTENSORA/DURAÇÃO:

TOMOU HOJE?

QUAL?

4. ANTECEDENTES PESSOAIS:

DifTeRia ( ), SífiLIS ( ), AMIGDALITE ( ), FeBRe ReUMÁtiCA ( ), ASMA ( ), ALERGiA A DROGA ( ), QUAL? CARDIOPATIA ( ) AVC ( ),

DIABETES MELLITUS ( ), NEFROPATIA ( ) EPIDEMIOLOGIA PARA ESQUISTOSSOMOSE ( ), EPIDEMIOLOGIA PARA DOENÇA DE CHAGAS ( ), CIRURGIA ( ) QUAL? TA ELEVADA: GESTAÇÃo ( ), PARTO ( ), PUERPÉRIO ( ), ANTICONCEPCIONAL ( ) ESTRESSE ( ) PROTESE ( )

LENTES CORRETORAS ( ), OUTROS:

5. VIDA SEXUAL ATIVA LIBIDO ALTERAÇÕES PÓS HIPOTENSOR ( ) QUAL?

6. ANTECEDENTES HEREDITÁRIOS:

CARDIOPATIA ( ) QUAL? QUEM? H.A.S. ( ) QUEM? A.V.C. ( ) QUEM? , DIABETES MELLITUS ( ) QUEM? , NEFROPATIA ( ) QUAL? QUEM? OUTROS: 


\section{ANEXO 2}

UNIVERSIDADE FEDERAL DE PERNAMBUCO HOSPITAL DAS CLÍNICAS SERVIÇO DE CARDIOLOGIA CLÍNICA DE HIPERTENSÃO ARTERIAL

\section{CONSULTA DE ENFERMAGEM}

7. HÁBITOS:

HIGIENE CORPORAL

ORAL SONO

TABAGISMO: TIPO

ETILISMO: TIPO , FUNÇÃODE ELIMINAÇÃO: DIURESE EXERCÍCIO FÍSICO QUANTIDADE QUANTIDADE

ALIMENTAÇÃO , EXERCICIO FISICO

$\longrightarrow$,

HIDRATAÇÃO , EVACUAÇÃO: LAZER
TEMPO TEMPO

8. EXAME FÍSICO

8.1 ESTADO GERAL:PESO___ g, ALTURA LOCOMOÇÃO cm, P. REFERÉNCIA (PROVISÓRIO) g,

8.2 ESTADO MENTAL: ORIENTADO ( ), CALMO ( ), ANSIOSO ( ), COMUNICATIVO ( ), OUTROS:

8.3 PELE E ANEXOS: COLORAÇÃO TURGORE ELASTICIDADE

PERFUSÃO TISSULAR PERIFÉRICA OUTROS:

8.4 APARELHO CARDIO-VASCULAR-RESPIRATÓRIO:

(10) PA ORTO: $X$ $\mathrm{mmHg}$

AUSCULTA CARDÍACA: RC

PULSOS RADIAIS/PEDIOSOS: RITMO FC

(2) PA ORTO: $x$ $\mathrm{mmHg}$ bPm, SOPRO ( ) NO FOCO:

CIRCULAÇÃO VENOSA PERIFÉRICA: CIANOSE ( ) AMPLITUDE , SIMETRIA ESTASE JUGULAR $45^{\circ}()$ AUSCULTA PULMONAR: M.V. RUIDOS ADVENTÍCIOS OUTROS:

8.5 ABDOMEN: NORMAL ( ), PANICULO ADIPOSO DOLOROSO ( ) DISTENDIDO ( ), VISCEROMEGALIA ( ), OUTROS:

9. PROBLEMAS IDENTIFICADOS:

PA ELEVADA ( ) ESTRESSE ( ) TABAGISMO ( ) HIPERCOLESTEROLEMIA ( ) ETILISMO ( ) SEDENTARISMO ( ) DIABETES MELLITUS ( ) OBESIDADE ( ) OUTROS:

10. CONDUTAS: ORIENTAÇÕES:

\section{ENCAMINHAMENTOS:}

EXAMES DE ROTINA:

RETORNO EM 1 ASS. 


\section{ANEXO 3}

UNIVERSIDADE FEDERAL DE PERNAMBUCO

HOSPITAL DAS CLÍNICAS - CLÍNICA DE HIPERTENSÃO - SERVIÇO DE CARDIOLOGIA

FICHA DE CONTROLE DO HIPERTENSO

NOME

REG. HAS

SEXO IDADE COR NATURALIDADE

INSTRUÇÃO OCUPAÇÃO ESTADO CIVIL

ENDEREÇO

RELIGIÃO PESO

ALTURA RETORNO

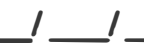

\begin{tabular}{l|l|l|l|l}
\hline DATA & PA/RC/FC & TRATAMENTO & PROBLEMAS & CONDUTA \\
\hline & & & & \\
& & & & \\
& & & &
\end{tabular}

